Pure Appl. Chem., Vol. 72, Nos. 1-2, pp. 189-197, 2000.

(C) 2000 IUPAC

\title{
Photophysics and spectroscopy of metal particles*
}

\author{
José H. Hodak, ${ }^{1}$ Arnim Henglein, ${ }^{2}$ and Gregory V. Hartland ${ }^{1}$ \\ ${ }^{1}$ Department of Chemistry and Biochemistry and ${ }^{2}$ Radiation Laboratory, \\ University of Notre Dame, Notre Dame, Indiana 46556-5670, USA
}

\begin{abstract}
This paper describes our recent work using ultrafast laser spectroscopy to examine the fundamental properties of metal particles. Two studies are presented. First, the characteristic time scale for electron-phonon coupling in Au particles with sizes between 2 and $120 \mathrm{~nm}$ has been examined by bleach recovery measurements. These experiments show that the coupling between the electrons and phonons is independent of particle size, to within the signal-tonoise of our experiments. We have also used transient absorption spectroscopy to examine the low-frequency "breathing" modes of the Au particles. These modes are impulsively excited by the rapid lattice heating that accompanies electron-phonon coupling. The breathing motion contributes to the transient absorption signal because the position of the plasmon band depends on the free electron density and, thus, the particle volume. The measured frequencies are inversely proportional to the radius, and almost exactly match the predictions of classical mechanics calculations for Au.
\end{abstract}

\section{INTRODUCTION}

Understanding how the properties of materials change with their size has been a long-standing goal in physical chemistry $[1,2]$. For example, an issue that has recently received considerable attention is whether the surface plays any role in mediating the coupling between the electrons and phonons in nanometer-sized metal particles [3-13]. The electron-phonon coupling constant can be measured by transient absorption spectroscopy. In these experiments a pump laser pulse excites a "hand-full" of electrons. The excited electrons subsequently undergo rapid electron-electron scattering (10-100 fs), which creates a hot electron distribution (temperature rises of several thousand $\mathrm{K}$ are possible). The hot electron distribution relaxes on a picosecond time scale by coupling to the lattice; this relaxation process can be monitored by a second probe laser pulse. Recent measurements for Sn [9] and Ag [12] particles have shown that the time scale for electron-phonon coupling decreases as the particle size decreases. However, for Au particles the size dependence of the electronic relaxation is a matter of some debate $[5-8,10,11,13]$. Much of this controversy is due to the fact that the measured decay time depends on the pump laser power: high-intensity pump pulses give high initial electronic temperatures and long relaxation times $[5,6,10]$. In this paper we present a study of the power and size dependence of the electronphonon coupling for Au particles in aqueous solution. The results show that for Au there is no size dependence in the coupling between the electrons and phonons (to within our signal-to-noise) [14].

At high pump laser power, the energy exchange between the electrons and phonons results in a substantial increase in the lattice temperature $(10-100 \mathrm{~K})$ and, therefore, lattice expansion. For $\mathrm{Au}$ particles larger than ca. $8 \mathrm{~nm}$ the time scale for heating the lattice is faster than the lowest-frequency acoustic phonon mode of the particles [15-17]. Thus, the rapid heating/expansion process can impulsively excite the symmetric breathing modes [17]. These modes change the particle size, which shifts the plasmon band because the peak position depends on the free electron density [15]. This affects both

*Pure Appl. Chem. 72, 1-331 (2000). An issue of reviews and research papers based on lectures presented at the $1^{\text {st }}$ IUPAC Workshop on Advanced Materials (WAM1), Hong Kong, July 1999, on the theme of nanostructured systems. 
the transmission and reflection of light at frequencies near the plasmon resonance, causing a modulation in transient absorption [15-17] or transient reflectivity measurements [18]. The measured frequencies for our samples closely follow the predictions of classical mechanics calculations [19]. The modulations decay after ca. 5-6 periods due to the polydispersity in the samples (different-sized particles have different modulation frequencies). By assuming a functional form for the size distribution, the average radius and width of the size distribution can be found by fitting the transient absorption data. The advantages (and disadvantages) of these experiments compared to transmission electron microscopy (the standard way of measuring the size of metal particles) are discussed.

\section{EXPERIMENTAL}

The transient absorption apparatus has been described elsewhere [11]. Briefly, the output of a regeneratively amplified Ti-sapphire laser (Clark-MXR, CPA-1000) is split to provide the pump and probe beams. The pump travels through a variable optical delay line before being frequency-doubled in a 1-mm BBO crystal. The probe is focussed into a 6-mm BK7 window or a 1-cm cuvette of water to generate a white light continuum. An interference filter selects different 10-nm portions of the white light continuum. The pump and probe beams had parallel polarizations and were overlapped at the sample by focussing with a $10-\mathrm{cm}$ lens. The transmitted probe is monitored by a photodiode (ThorLabs, PDA150) and a boxcar integrator (Stanford Research, SR250). A separate photodiode-boxcar combination detects a reference beam, which is split off from the probe before the sample. The output from the signal boxcar is divided by the reference with an analog divider. A lock-in amplifier (Stanford Research, SR830), referenced to a synchronous chopper (Stanford Research, SR540) monitors the output of the divider. The pump beam intensity was adjusted with a waveplate-polarizer combination. The pump laser powers used were $0.01-0.3 \mu \mathrm{J} /$ pulse for the electron-phonon coupling measurements, and $1-5 \mu \mathrm{J} /$ pulse for the acoustic modulation experiments. Cross-correlations of the pump beam with the selected 10-nm bandwidth portions of the white light continuum typically had full-widths-at-half-maximum of

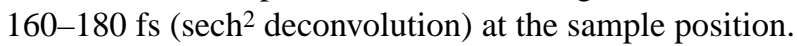

Nanoparticles of gold of any desired size and a rather narrow size distribution were prepared by growing smaller seed particles, as recently described [20]. The growth of the gold seed particles is radiolytically controlled as follows: a solution of the seed particles containing $0.2 \mathrm{M}$ methanol and potassium dicyano-aurate-I is $\gamma$-irradiated $\left(9 \times 10^{4} \mathrm{rad} / \mathrm{h}\right)$ under an atmosphere of nitrous oxide. This produces reducing hydroxymethyl radicals, which cathodically charge the particles via electron transfer. The stored electrons directly reduce dicyanogold on the particle surface. The irradiation is carried out until all the dicyanogold is reduced. A great advantage of this method is that the size distribution of the final sample is substantially narrower than that of the seeds [21], an important requirement for the laser experiments reported here. Two types of seed particles were used. To prepare large particles, 15-nm-diameter seeds were made via citrate reduction of tetrachloroaurate-III [22]. For smaller particles, 2-nm-diameter seeds were made by $\gamma$-irradiating a deaerated $2 \times 10^{-4} \mathrm{M} \mathrm{NaAuCl}_{4}$ solution containing $10^{-2} \mathrm{M}$ polvinylalcohol and $0.2 \mathrm{M}$ methanol [21]. The required amount of $\mathrm{Au}(\mathrm{CN})_{2}{ }^{-}$was then added and the $\gamma$-irradiation continued until all the additive was reduced. The final particle solutions contained cyanide ions from the reduction of $\mathrm{Au}(\mathrm{CN})_{2}^{-}$, which were removed by adding ion exchange resin (amberlite MB 150 from Aldrich) and decanting after slight shaking for 1 hour. The colloidal solutions were then stable under air for months. Particle size distributions were determined from transmission electron micrographs (Hitachi EM, type $\mathrm{H} 600,80 \mathrm{kV}$ ). The preparation of the $\mathrm{Cu}$ particles has been described elsewhere [23]. This procedure consists of the radiolytic reduction of dicyano-copprate$\mathrm{I}, \mathrm{Cu}(\mathrm{CN})_{2}{ }^{-}$. The deaerated aqueous solution contained $4 \times 10^{-4} \mathrm{M} \mathrm{KCu}(\mathrm{CN})_{2}, 0.5$ to $1.3 \times 10^{-4} \mathrm{M}$ excess $\mathrm{KCN}$, and $0.3 \mathrm{M}$ 2-propanol. A slow reduction occurred upon $\gamma$-irradiation, and the size of the particles formed became smaller with decreasing excess $\mathrm{KCN}$ and increasing dose rate. The $\mathrm{Cu}$ par- 
ticles produced were less uniform in size and shape than the Au particles described above. This can be seen from Fig. 6 below, which shows large polyhedra, smaller spheres, and rod-like structures.

\section{RESULTS AND DISCUSSION}

\section{Electron-Phonon Coupling}

Figure 1 shows the change in intensity of the transmitted probe light, as a function of time and wavelength for a sample of 8-nm-diameter Au particles after 390-nm pump laser excitation. The most intense feature is a strong bleach (an increase in transmitted intensity due to a decrease in the sample absorbance) at the peak of the plasmon band (ca. $525 \mathrm{~nm}$ for this sample). Note that the signal changes to an absorbance (a decrease in transmission) near $600 \mathrm{~nm}$. These features arise because the plasmon band becomes broader when the electrons are heated by the pump laser pulse [5,10]. Figure 2 shows calculated spectra for Au particles at two different electronic temperatures $(298 \mathrm{~K}$ and $1000 \mathrm{~K})$. In these calculations the temperature-dependent dielectric constant is obtained using the theory of Rosei et al. [24], and the absorbance cross-section of the particles was calculated using the Mie equation [25]. Note that all the temperature dependence is assigned to the interband transitions of the electrons in this model [11]. The calculations clearly show that increasing the electronic temperature broadens the plasmon band, creating a transient bleach signal at the band maximum and an absorbance signal in the wings of the band. Both these features decay as the electrons equilibrate with the lattice via electron-phonon coupling.

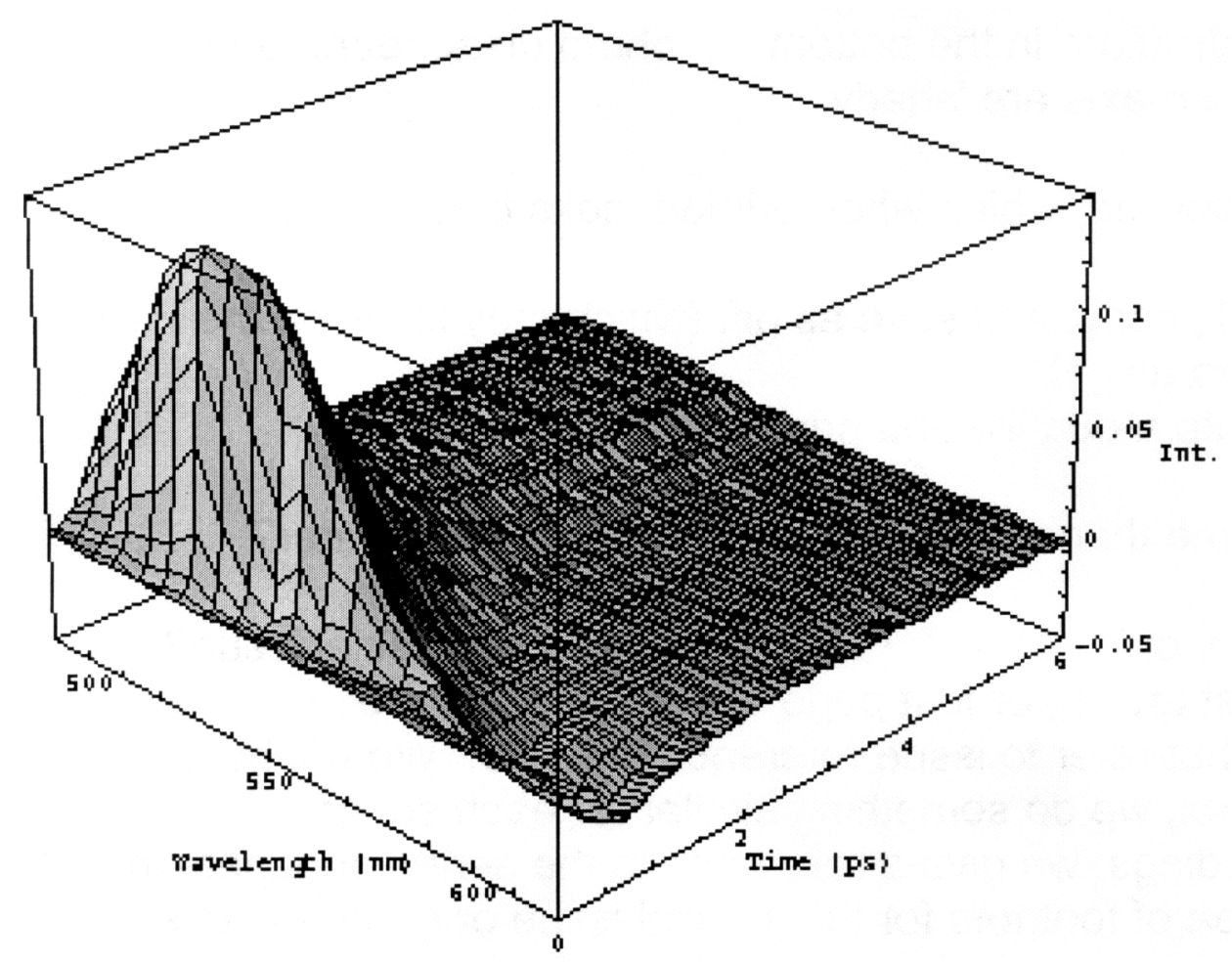

Fig. 1 Change in transmission for 8-nm-diameter Au particles as a function of time after 390-nm pump laser excitation. The different wavelength traces were recorded by scanning a monochromator after the sample.

(C) 2000 IUPAC, Pure and Applied Chemistry 72, 189-197 


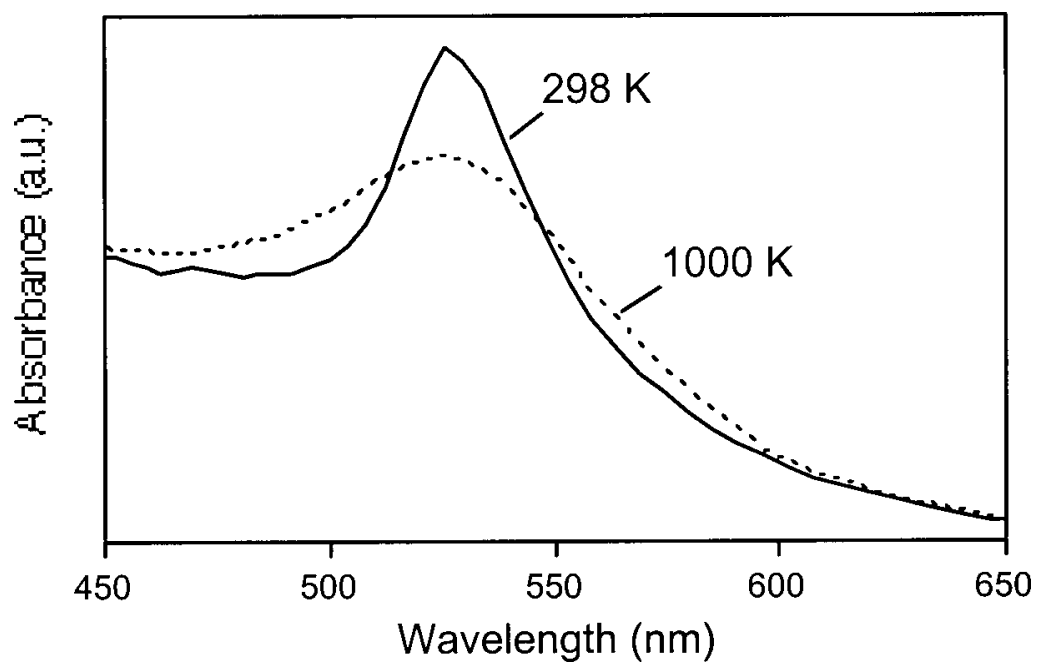

Fig. 2 Calculated spectra for Au particles in aqueous solution for two different electronic temperatures. Details of these calculations can be found in Ref. [11].

Traces recorded at any single wavelength can show a complicated time dependence. For example, in the 550 to $600 \mathrm{~nm}$ region, the signal changes from a bleach to an absorption as time progresses. Clearly this makes it difficult to interpret the data in terms of a decay of the electronic temperature. Calculations show that the signal is proportional to electronic temperature for probe wavelengths near the peak of the plasmon band $(520-530 \mathrm{~nm})$, or for probe wavelengths $\lambda>600 \mathrm{~nm}$. Of these two options, it is better to perform experiments with green probe pulses, as the signals are much larger (see Fig. 1) and, therefore, much higher sensitivity can be obtained. This is important because accurate measurements of the electron-phonon coupling constant require low pump laser powers. Indeed, differences in the pump laser power probably account for most of the differences in the results obtained for Au particles by different groups [5-8,10,11,13,14].

Figure 3 shows the time constant for the decay of the electronic temperature as a function of relative pump laser intensity $\left(I / I_{o}\right)$ for an 8-nm Au particle sample. These experiments were performed with 390-nm pump pulses and with the probe laser tuned to the peak of the plasmon band. The time

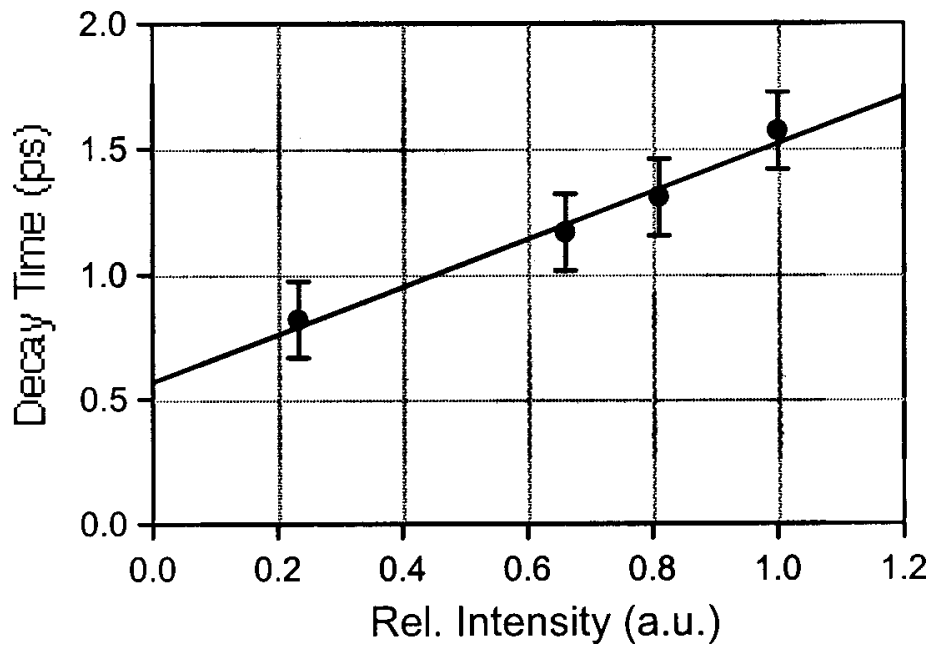

Fig 3 Experimentally determined electron-phonon relaxation times for 8-nm-diameter Au particles versus relative pump laser power. The experiments were performed with 390-nm pump and 530-nm probe pulses. Also shown is a straight line fit to the data that yields an intercept of $0.6 \pm 0.1 \mathrm{ps}$. 
constants were determined by fitting the transient bleach data to an exponential decay function. The decay times obtained vary from $<1 \mathrm{ps}$ to $\sim 2 \mathrm{ps}$ as the pump laser power increases. This behavior can be explained by the two-temperature model for electron-phonon coupling: higher pump laser powers yield higher initial electronic temperatures and, therefore, longer decay times due to the temperature dependence of the electronic heat capacity $[26,27]$. According to the two-temperature model, the intercept where $I=0$ is given by $\gamma T_{\mathrm{o}} / g$, where $\gamma T_{\mathrm{o}}$ is the electronic heat capacity at room temperature and $g$ is the electron-phonon coupling constant $[10,11]$. The intercept in Fig. $3(0.6 \pm 0.1 \mathrm{ps})$ yields an electronphonon coupling constant of $g=(3.3 \pm 0.5) \times 10^{16} \mathrm{~W} \mathrm{~m}^{-3} \mathrm{~K}^{-1}$, which is the same as that for bulk $\mathrm{Au}$ [27]. Measurements for different-sized particles show that the value of the electron-phonon coupling constant does not change with size (to within our signal-to-noise) for particles as small as $2.5 \mathrm{~nm}$ diameter [14]. This is a surprising result: the electron mean free path in Au is 30-40 nm, thus, the electrons must scatter off the surface for these very small particles. However, the fact that the electronphonon coupling constant does not change with size shows that these scattering events are elastic [1114].

\section{Impulsive Lattice Heating}

The results in the previous section show that the time scale for the decay of the electronic temperature is on the order of ps for nanometer-sized Au particles (the exact value depends on the pump laser intensity). As the electronic temperature decays there is a concomitant increase in the temperature of the lattice. This rise in the lattice temperature causes the particles to expand [11,15-17]. Specifically, the increase in size is $\Delta R / R=\alpha\left(T_{l}-298\right) / 3$, where $R$ is the radius, $T_{l}$ is the lattice temperature and $\alpha$ is the coefficient for thermal expansion of Au. For Au particles larger than ca. $8 \mathrm{~nm}$, the increase in the lattice temperature is faster than the lowest-frequency acoustic phonon mode of the particles [15-17]. Thus, the lattice cannot instantaneously respond to the temperature increase. This creates a situation where the acoustic phonon modes can be impulsively excited. Note that because the lattice is heated isotropically we only expect symmetric "breathing" modes to be excited [17]. An example of a transient absorption experiment where the coherently excited phonon modes have been observed for 60-nm-diameter $\mathrm{Au}$

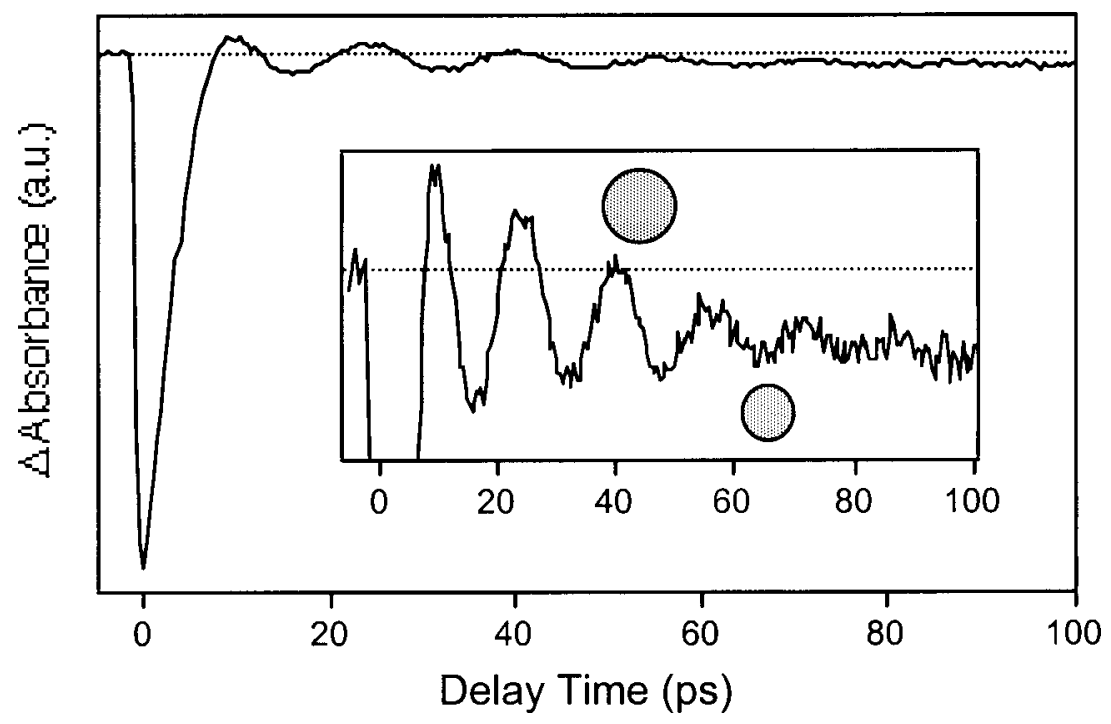

Fig. 4 Transient absorption data for 60-nm-diameter Au particles recorded with 390-nm pump and 550-nm probe laser pulses. The insert shows an expanded view of the oscillating part of the signal.

(C) 2000 IUPAC, Pure and Applied Chemistry 72, 189-197 
particles is shown in Fig. 4. The insert in Fig. 4 is simply a "blow-up" of the oscillating part of the signal. This experiment was performed with 390-nm pump laser pulses and 550-nm probe laser pulses (i.e., the signal was recorded on the red side of the plasmon band).

The coherently excited breathing modes contribute to the transient absorption signal because they change the particle size. This changes the free electron density, which shifts the plasmon band [28]. To understand qualitatively how this arises, note that the frequency of the plasmon band maximum can be expressed as $\omega_{\max }=\omega_{p} /\left(1+2 \varepsilon_{m}\right)^{1 / 2}$, where $\varepsilon_{m}$ is the dielectric constant of the medium and $\omega_{p}$ is the plasma frequency. The plasma frequency is a property of the metal and is given by $\left(n e^{2} / \varepsilon_{o} m_{e}\right)^{1 / 2}$, where $n$ is the free electron density [25]. Thus, an increase in size will decrease $n$ and red-shift the plasmon band. This causes a transient absorption signal when the probe is tuned to the red of the plasmon band (this is shown schematically in Fig. 4).

Figure 5 shows how the frequency of the oscillations depends on the particle size. The experimental frequencies were obtained by fitting the data to a damped cosine function and the size distributions were measured by TEM. The frequency is proportional to $1 / R$, and a straight line fit yields $v\left(\mathrm{~cm}^{-1}\right)=$ $(50.75 \pm 0.95) / R$, for $R$ in $\mathrm{nm}$. From classical mechanics calculations, the frequency of the breathing mode of a sphere is $v\left(\mathrm{~cm}^{-1}\right)=\left(\eta c_{l}\right) /(2 \pi c R)$, where $c_{l}$ is the longitudinal speed of sound in Au, $c$ is the speed of light, and $\eta$ is an eigenvalue that depends on the ratio of the longitudinal and transverse speeds of sound [19,29]. For Au this eigenvalue is $\eta=2.93$, in comparison our data yields $\eta=2.95 \pm 0.06$. The

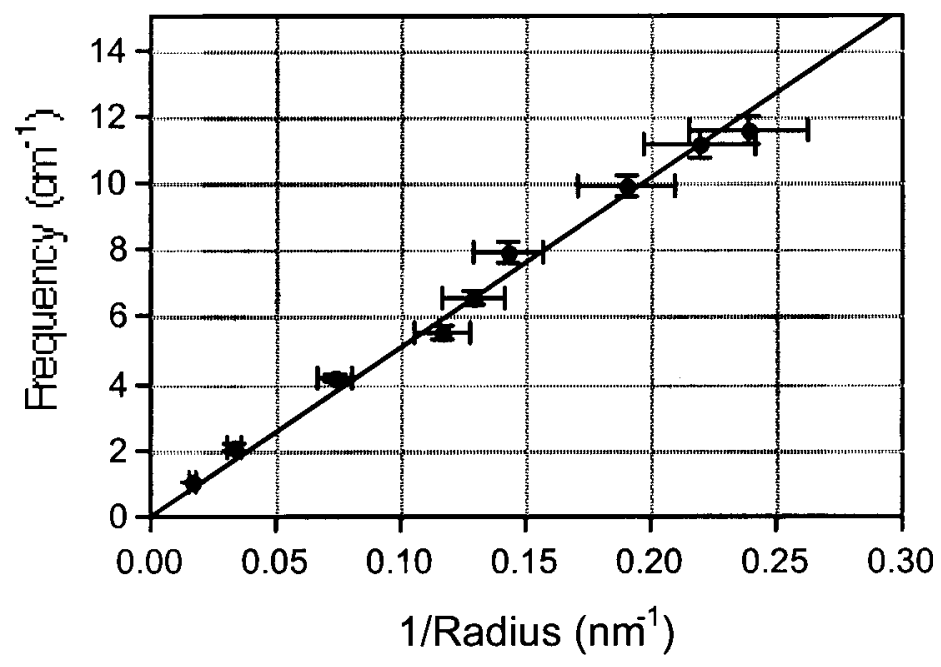

Fig. 5 Frequency of the acoustic breathing mode for Au versus the inverse of the particle radius. The particle sizes (mean radius and width) were determined by TEM, and the frequencies were obtained by fitting the transient absorption data to a damped cosine function. The straight line is a fit to the data.

excellent agreement between the predicted and experimental frequencies confirms the assignment of the modulation as due to the acoustic breathing mode [16,17].

The inverse dependence of the breathing mode frequency on the particle radius means that the modulations will be inhomogenously damped for polydisperse samples. Calculations of the expected decay using the particle size distribution measured by TEM show that this damping mechanism dominates for metal particles in aqueous solution [16]. Thus, these experiments cannot be used to obtain information about the intrinsic dephasing of the low-frequency phonon modes. On the other hand, the frequency and decay of the modulations could be used to determine the size distribution of the sample, as long as the transverse and longitudinal speeds of sound are known for the particles. The excellent 
match between the calculated and experimental frequencies shown in Fig. 5 indicates that this procedure should work well for spherical particles.

An example of a transient absorption experiment for a $\mathrm{Cu}$ particle sample is shown in Fig. 6(a). The calculated curve was obtained from a sum of cosine functions $A_{i} \cos \left(\left(\eta c_{l} / R_{i}\right) t+\phi\right)$, where the weights $A_{i}$ were calculated by assuming a normal distribution for the particle radii $R_{i}$, and $\eta$ and $c_{l}$ were determined from data in the CRC Handbook. A least squares fitting routine was used to adjust the average radius $\langle R\rangle$ and width $\sigma$ of the size distribution to best fit the data. The results are given in the figure. Note that the first period of the oscillation is not well fitted because it is distorted by the transient bleach signal from the hot electrons. Figure 6(b) shows a representative TEM picture of the same $\mathrm{Cu}$ particle sample. It can be clearly seen that the sample is polydisperse, and that the average radius from TEM (ca. $28 \mathrm{~nm}$ ) is smaller than that calculated from the transient absorption data (ca. $33 \mathrm{~nm}$ ). The Cu particles are also not spherical. The expected acoustic modes for non-spherical particles are extremely difficult to calculate. Numerical results for cubes show that the breathing mode frequencies are lower than those for spheres [30]. Thus, our analysis, which assumes spherical particles, would overestimate
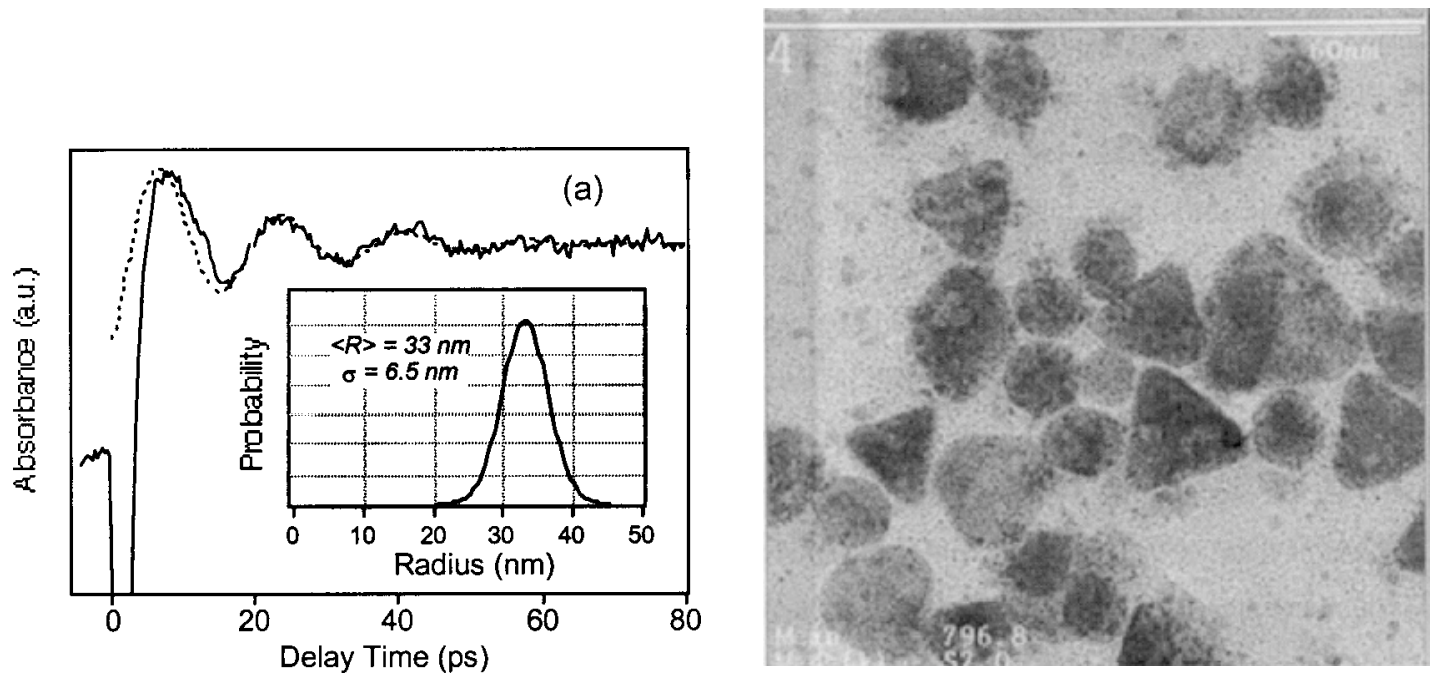

Fig. 6 (a) Left: Transient absorption data (solid line) recorded for $\mathrm{Cu}$ particles in aqueous solution. The pump and probe wavelengths were $390 \mathrm{~nm}$ and $600 \mathrm{~nm}$, respectively. Also shown is a fit to the data (dashed line) using a sum of cosine functions with frequencies and weights determined assuming a normal distribution of particle sizes. The size distribution derived from the data is shown in the insert. (b) Right: Representative TEM picture of the $\mathrm{Cu}$ particle sample used in (a). The scale is approximately $1 \mathrm{~cm}=30 \mathrm{~nm}$.

the size for non-spherical particles, which is indeed observed. Our experiments are also biased towards the larger particles in the sample, because these particles have larger absorption cross-sections [25].

Despite these problems, there are several advantages of this technique for measuring size distributions compared to TEM. First, because the method described above is an all optical technique it can be used to make measurements in situ. This is a big advantage for air sensitive or unstable samples, which might be destroyed by preparing a TEM grid. Indeed, the Cu particle samples shown in Fig. 6 rapidly dissolve in the presence of oxygen. Second, our transient absorption experiments interrogate $10^{4}-10^{8}$ particles, depending on the size of particles and the exact conditions used in the experiments. In contrast, TEM measurements interrogate $10-10^{2}$ particles. Thus, the results from the transient ab- 
sorption experiments are statistically much more reliable. The main disadvantages of this technique are that the results are not accurate for non-spherical particles, the average radius is biased towards the larger particles, and that the modulations in the transient absorption signal cannot be observed for very small particles. The last point arises because small particles have higher frequency breathing modes, and high frequency modes cannot be coherently excited by the relatively slow lattice heating process (impulsive excitation does not occur when the impulse has a similar time scale to the period of motion). For $\mathrm{Au}$, the modulations cannot be excited for particles smaller than ca. $8 \mathrm{~nm}$ [16]. For $\mathrm{Cu}$, this cut-off occurs at a larger size because the longitudinal speed of sound and, therefore, the frequencies of the breathing modes are larger for $\mathrm{Cu}$ than for $\mathrm{Au}$.

\section{SUMMARY AND CONCLUSIONS}

Excitation of nanometer-sized metal particles with an ultrafast laser pulse produces a rapid increase in the electronic temperature. The hot electron distribution equilibrates with the lattice via electron-phonon coupling on a picosecond time scale. The exact value of the relaxation time depends on the intensity of the pump laser: higher pump powers give higher initial temperatures and longer electron-phonon coupling times. At low pump laser power, the decay times are the same as that for bulk Au [27], and are similar for different-sized particles. Thus, particle size does not affect the coupling between the electrons and phonons, for Au particles as small as $2.5 \mathrm{~nm}$ diameter [14].

The energy exchange between the electrons and the phonons increases the temperature of the lattice, which causes the particles to expand. For Au particles with diameters larger than ca. $8 \mathrm{~nm}$, lattice heating occurs on a faster time scale than the lowest-frequency acoustic "breathing" mode of the particle. Thus, the acoustic breathing modes can be impulsively excited. The modulation in the particle volume caused by the coherently excited breathing motion produces a periodic change in the free electron density, which shifts the position of the plasmon band. This gives rise to a beat signal in transient absorption experiments. We have found that for gold particles in aqueous solution the frequency of the breathing mode is given by $v\left(\mathrm{~cm}^{-1}\right)=(2.95 \pm 0.06) c_{l} /(2 \pi c R)$ where $R$ is the particle radius, $c_{l}$ is the longitudinal speed of sound in $\mathrm{Au}$, and $c$ is the speed of light. This almost exactly matches the relationship expected from classical calculations that treat the particles as free elastic spheres [19,29]. Examination of the decay of the modulations shows that their lifetime is controlled by the distribution of particle sizes: different-sized particles oscillate with different frequencies, which causes a dephasing in the signal. Thus, these time-resolved experiments provide a convenient way of measuring the size distribution of metal particles in solution. Future research directions in our laboratory are to use these measurements to examine bi-metallic particles, both alloys and core-shell type particles, and to see whether we can detect lattice melting.

\section{ACKNOWLEDGMENTS}

The work described here was supported by the NSF, Grant \# CHE98-16164 (GVH and JHH) and by the Office of Basic Energy Sciences of the U.S. Department of Energy through the Notre Dame Radiation Laboratory $(\mathrm{AH})$. The authors are also grateful to Dr. Michael Giersig for performing the TEM measurements.

\section{REFERENCES}

1. A. Henglein. J. Phys Chem. 97, 5457 (1993).

2. A. P. Alivisatos, J. Phys Chem. 100, 13226 (1996).

3. J.-Y. Bigot, J.-C. Merle, O. Cregut, A. Daunois. Phys. Rev. Lett. 75, 4702 (1995). 
4. T. W. Roberti, B. A. Smith, J. Z. Zhang. J. Chem. Phys. 102, 3860 (1995).

5. T. S. Ahmadi, S. L. Logunov, M. A. El-Sayed. J. Phys. Chem. 100, 8053 (1996).

6. S. L. Logunov, T. S. Ahmadi, M. A. El-Sayed; J. T. Khoury, R. L. Whetten. J. Phys. Chem. B 101, 3713 (1997).

7. B. A. Smith, J. Z. Zhang, U. Griebel, G. Schmid. Chem. Phys. Lett. 270, 139 (1997).

8. M. Perner, P. Bost, U. Lemmer, G. van Plessen, J. Feldmann, U. Becker, M. Mennig, M. Schmitt, H. Schmidt. Phys. Rev. Lett. 78, 2192 (1997).

9. M. Nisoli, S. Stagira, S. De Silvestri, A. Stella, P. Tognini, P. Cheyssac, R. Kofman. Phys. Rev. Lett. 78, 3575 (1997).

10. J. H. Hodak, I. Martini, G. V. Hartland. Chem. Phys. Lett. 284, 135 (1998).

11. J. H. Hodak, I. Martini, G. V. Hartland. J. Phys. Chem. B 102, 6958 (1998).

12. N. Del Fatti, C. Flytzanis, F. Vallée. Appl. Phys. B. 68, 433 (1999).

13. S. Link, C. Burda, Z. L. Wang, M. A. El-Sayed. J. Chem. Phys. 111, 1255 (1999).

14. J. H. Hodak, A. Henglein, G. V. Hartland. J. Chem. Phys. 112, 5942 (2000).

15. J. H. Hodak, I. Martini, G. V. Hartland. J. Chem. Phys. 108, 9210 (1998).

16. J. H. Hodak, A. Henglein, G. V. Hartland. J. Chem. Phys., 111, 8613 (1999).

17. N. Del Fatti, C. Voisin, F. Chevy, F. Vallée, C. Flytzanis. J. Chem. Phys. 110, 11,484 (1999).

18. M. Nisoli, S. De Silvestri, A. Caralleri, A. M. Malvezzi, A. Stella, G. Lanzani, P. Cheyssac, R. Kofman. Phys. Rev. B 55, R13424 (1997).

19. H. Lamb. Proc. Lond. Math. Soc. 13, 189 (1882).

20. A. Henglein and D. Meisel. Langmuir 14, 7392 (1998).

21. A. Henglein. Langmuir, 15, 6738 (1999).

22. B. V. Enustun and J. Turkevich. J. Am. Chem. Soc. 85, 3317 (1963).

23. A. Henglein. J. Phys. Chem. B 104, 1206 (2000).

24. R. Rosei, F. Antonangeli, U. M. Grassano. Surf. Sci. 37, 689 (1973).

25. U. Kriebig and M.Vollmer. Optical Properties of Metal Clusters, Springer, Berlin (1995).

26. C.-K. Sun, F. Vallée, L. H. Acioli, E. P. Ippen, J. G. Fujimoto. Phys. Rev. B 50, 15337 (1994).

27. R. H. M. Groeneveld, R. Sprik, A. Lagendijk. Phys. Rev. B 51, 11433 (1995).

28. P. Mulvaney. Langmuir 12, 788 (1996).

29. V. A. Dubrovskiy and V. S. Morochnik. Izv. Earth Phys. 17, 494 (1981).

30. J. Zhao, Y. Masumoto. Phys. Rev. B 60, 4481 (1999).

(C) 2000 IUPAC, Pure and Applied Chemistry 72, 189-197 